\title{
Clinical Microbiology
}

National Cancer Institute

\section{Source}

National Cancer Institute. Clinical Microbiology. NCI Thesaurus. Code C18702.

The discipline focusing on microbe-induced clinical conditions, including epidemiology, pathogenesis, diagnosis and detection, and prevention and treatment. 